\title{
Continuous quality improvement program and its results of Korean Society for Cytopathology
}

\author{
Yoo-Duk Choi ${ }^{1,2}$, Hoon-Kyu Oh ${ }^{1,3}$, Su-Jin Kim ${ }^{1,4}$, Kyung-Hee Kim ${ }^{1,5}$, Yun-Kyung Lee ${ }^{1,6}$, Bo-Sung Kim ${ }^{1,7}$, Eun-Jeong Jang ${ }^{1,8}$, \\ Yoon-Jung Choi ${ }^{1,9}$, Eun-Kyung Han ${ }^{1,10}$, Dong-Hoon Kim ${ }^{1,11}$, Younghee Choi ${ }^{1,12}$, Chan-Kwon Jung ${ }^{1,13}$, Sung-Nam Kim ${ }^{1,14}$, \\ Kyueng-Whan Min ${ }^{1,15}$, Seok-Jin Yoon ${ }^{1,16}$, Hun-Kyung Lee ${ }^{1,17}$, Kyung Un Choi ${ }^{1,18}$, Hye Kyoung Yoon ${ }^{1,19}$ \\ ${ }^{1}$ The Committee of Quality Improvement of Korean Society for Cytopathology, Seoul; '2Department of Pathology, Chonnam National University Medical School, Gwangju; \\ ${ }^{3}$ Department of Pathology, Daegu Catholic University School of Medicine, Daegu; ' $D$ Department of Pathology, Seegene Medical Foundation, Busan; \\ ${ }^{5}$ Department of Pathology, Chungnam National University School of Medicine, Daejeon; \\ ${ }^{6}$ Department of Pathology and Translational Genomics, Samsung Medical Center, Sungkyunkwan University School of Medicine, Seoul; \\ ${ }^{7}$ Department of Pathology, National Medical Center, Seoul; ${ }^{8}$ Department of Pathology, Fatima Hospital, Daegu; \\ ${ }^{9}$ Department of Pathology, National Health Insurance Service, Ilsan Hospital, Goyang; ${ }^{10}$ Department of Pathology, Seoul Clinical Laboratories, Seoul; \\ ${ }^{11}$ Department of Pathology, Kangbuk Samsung Hospital, Sungkyunkwan University School of Medicine, Seoul; \\ ${ }^{12}$ Department of Pathology, Hallym University Dongtan Sacred Heart Hospital, Hwaseong; \\ ${ }^{13}$ Department of Hospital Pathology, College of Medicine, The Catholic University of Korea, Seoul; ${ }^{14}$ Department of Pathology, Sure Quest Lab, Yongin; \\ ${ }^{15}$ Department of Pathology, Hanyang University Guri Hospital, Hanyang University College of Medicine, Guri; \\ ${ }^{16}$ Department of pathology, Dankook University Hospital, Cheonan; ${ }^{77}$ Department of Pathology, Ewha Laboratory of Medicine, Seoul; \\ ${ }^{18}$ Department of Pathology, Pusan National University Hospital, Busan; ${ }^{19}$ Department of Pathology, Busan Paik Hospital, Inje University, Busan, Korea
}

\begin{abstract}
Background: Since 1995, the Korean Society for Cytopathology has overseen the Continuous Quality Improvement program for cytopathology laboratories. The Committee of Quality Improvement has carried out an annual survey of cytology data for each laboratory and set standards for proficiency tests. Methods: Evaluations were conducted four times per year from 2008 to 2018 and comprised statistics regarding cytology diagnoses of previous years, proficiency tests using cytology slides provided by the committee, assessment of adequacy of gynecology (GYN) cytology slides, and submission of cytology slides for proficiency tests. Results: A total of 206 institutes participated in 2017, and the results were as follows. The number of cytology tests increased from year to year. The ratio of liquid-based cytology in GYN gradually decreased, as most of the GYN cytology had been performed at commercial laboratories. The distribution of GYN diagnoses demonstrated nearly $3.0 \%$ as atypical squamous cells. The rate for squamous cell carcinoma was less than $0.02 \%$. The atypical squamous cell/squamous intraepithelial lesion ratio was about 3:1 and showed an upward trend. The major discordant rate of cytology-histology in GYN cytology was less than $1 \%$. The proficiency test maintained a major discordant rate less than $2 \%$. The rate of inappropriate specimens for GYN cytology slides gradually decreased. Conclusions: The Continuous Quality Improvement program should be included in quality assurance programs. Moreover, these data can contribute to development of national cancer examination guidelines and facilitate cancer prevention and treatment.
\end{abstract}

Key Words: Cytology; Quality; Statistics; Proficiency

Received: December 27, 2019 Revised: February 16, 2020 Accepted: February 22, 2020

Corresponding Author: Hoon-Kyu Oh, MD, PhD, Department of Pathology, Daegu Catholic University School of Medicine, 33 Duryugongwon-ro 17-gil, Namgu, Daegu 42472, Korea Tel: +82-53-650-4156, Fax: +82-53-650-3456, E-mail: ap510@cu.ac.kr

The Committee of Quality Improvement of the Korean Society for Cytopathology (CQIKSC) was organized in 1992 for quality control management in advancement of cytopathology. In the initial period, the Committee distributed quality assurance slides to participating institutions, assessed diagnostic accuracy based on the received results, and issued quality assurance certification to those who qualified. Since 2004, the Committee has collected internal quality assurance data for the previous year from each institution at the first of the four quality assurance evaluation rounds per year and evaluated diagnostic accuracy from the second to fourth rounds. In 2008, the Committee compiled and reported the quality assurance status for the cytopathology field until 2007 in Korean Journal of Cytopatbology [1].

This study seeks to explore future quality assurance in cyto- 
pathology while simultaneously evaluating past work by compiling and reporting the results of cytopathologic quality assurance situations and activities.

\section{MATERIALS AND METHODS}

This study was based on data collected from 2008 to 2018 by institutions participating in cytopathologic quality assurance. Data collected included: (1) previous years' statistics of internal quality control (data from 2007 to 2017), including degree of concordance between gynecology (GYN) cytology and histology correlation; (2) proficiency test of cytology slides provided by the CQIKSC; (3) assessment of adequacy of GYN cytology slides; and (4) cytology slides submitted for proficiency tests. For this study, the institutions were categorized into three groups: university hospitals, general hospitals, and commercial laboratories [2].

\section{Statistics of internal quality control}

The first quality assurance implementation of the year was to collect internal quality control data of the previous year, including GYN, non-GYN, fine needle aspirations (FNA) cytology statistics, test methods, diagnosis-specific frequencies (under the Bethesda assessment criteria for GYN cytology), statistics on concordance between GYN cytology and histology correlation, personnel situations of cytopathologists, and initial microscopic specimen assessments. GYN cytology methods were investigated using conventional smear method or liquid-based cytology (LBC). Concordance between GYN cytology and histology was analyzed based on the criteria defined by the CQIKSC. Briefly, the results were categorized into category $\mathrm{O}$, concordant; category A, discordant but minimal clinical impact; category $\mathrm{B}$, discordant with minor clinical impact; and category $\mathrm{C}$, discordant with major clinical impact.

\section{Proficiency test for cytology slides provided by the CQIKSC} (external quality control)

The slides for proficiency tests were provided every year by institutions participating in quality assurance. The slides were randomly sent to the institutions according to an ordered series, and each institution returned their diagnostic results along with an evaluation of the adequacy of the slides received. Any slides that were deemed inadequate were reexamined by the CQIKSC and reevaluated for appropriateness as quality assurance slides. From 2008 to 2012, virtual slide or image file-based proficiency tests were also conducted once a year. However, since 2013, only the slide-based proficiency test has been implemented annually (Table 1).

For proficiency tests, a total of five slides was sent to each institution, consisting of two GYN slides, one non-GYN slide (body fluid or urine), and two respiratory sample or FNA slides. The diagnoses received by each institution within a set period were compared by CQIKSC. With respect to the diagnostic form, the cervical smear test was evaluated based on the Bethesda system [3], and the thyroid gland FNA test was based on the Bethesda

Table 1. Changes in quality improvement items from 2007 to 2017

\begin{tabular}{|c|c|c|c|c|}
\hline & First & Second & Third & Fourth \\
\hline 2007 & $\begin{array}{l}\text { Total C. statistics } \\
\text { Diagnostic-specific frequency of GYN C. } \\
\text { Concordance between GYN C. and H. }\end{array}$ & Proficiency test (3 slides) & Proficiency test (3 slides) & Proficiency test (3 slides) \\
\hline 2008 & $\begin{array}{l}\text { Total C. statistics } \\
\text { Diagnostic-specific frequency of GYN C. } \\
\text { Concordance between GYN C. and H. }\end{array}$ & Proficiency test (5 slides) & Proficiency test (virtual slide) & - \\
\hline 2009 & $\begin{array}{l}\text { Total C. statistics } \\
\text { Diagnostic-specific frequency of GYN C. } \\
\text { Concordance between GYN C. and H. }\end{array}$ & Proficiency test (5 slides) & Proficiency Test (5 image files) & $\begin{array}{l}\text { Slide submission } \\
\text { Image submission }\end{array}$ \\
\hline 2010 & $\begin{array}{l}\text { Total C. statistics } \\
\text { Diagnostic-specific frequency of GYN C. } \\
\text { Concordance between GYN C. and H. } \\
\text { Describe the discordant GYN cases }\end{array}$ & Proficiency test (5 image files) & Proficiency test (5 slides) & $\begin{array}{l}\text { Slide submission } \\
\text { Image submission }\end{array}$ \\
\hline $2011-12$ & $\begin{array}{l}\text { Total C. statistics } \\
\text { Diagnostic-specific frequency of GYN C. } \\
\text { Concordance between GYN C. and H. } \\
\text { Describe the discordant GYN cases }\end{array}$ & Proficiency test (5 slides) & Proficiency test (4 image files) & Slide submission (6 slides) \\
\hline $2013-17$ & $\begin{array}{l}\text { Total C. statistics } \\
\text { Diagnostic-specific frequency of GYN C. } \\
\text { Concordance between GYN C. and H. } \\
\text { Describe the discordant GYN cases }\end{array}$ & Proficiency test (5 slides) & $\begin{array}{l}\text { Assessment of adequacy for } \\
\text { GYN } 5 \text { slides }\end{array}$ & Slide submission (6 slides) \\
\hline
\end{tabular}

GYN, gynecology; C., cytology; H., histology. 
system for reporting thyroid cytopathology [4]. The CQIKSC defined the diagnostic forms of respiratory sample, body fluid, and FNA for organs other than the thyroid gland In addition, the Committee set up the evaluation criteria for diagnostic concordance for GYN, non-GYN, and FNA tests, classifying them into category $\mathrm{O}$, concordant; category $\mathrm{A}$, discordant but minimal clinical impact; category B, discordant with minor clinical impact; and category $\mathrm{C}$, discordant with major clinical impact. Institutions that were categorized into $C$ for any slide were scheduled to receive one reevaluation. If still categorized into $C$ in the reevaluation, the institution did not receive the quality assurance certification for the corresponding round and was required to undergo cytopathologic training. Repeated category $\mathrm{C}$ also indicated failure in the cytopathology area of the proficiency evaluation of the Quality Assurance of Pathology Division at the Korean Society of Pathologists.

\section{Assessment of adequacy of gynecologic cytology slides}

Since 2013, GYN slide adequacy has been evaluated in the Cytology Quality Improvement program once a year. Institutions evaluate the adequacy of five consecutive gynecologic slides prepared on a given random day and send the slides and reports to CQIKSC, which then assesses the adequacy of the GYN sample. Using this method, each institution was evaluated to ensure that the adequacy of samples had been properly reported.

\section{Cytology slide submission for diagnostic accuracy evaluation}

Every year, institutions participating in quality assurance submitted to the CQIKSC two GYN slides, two non-GYN slides, and two FNA slides for proficiency tests. The submitted slides were limited to cases of histologically confirmed diagnoses. CQIKSC members reviewed the slides to ensure that they were appropriate and useful as quality assurance data before using them as quality control slides.

\section{Statistics}

The distribution of GYN diagnostic categories according to year was conducted using the score test for trend in IBM SPSS Statistics software ver. 23.0 (IBM Corp., Armonk, NY, USA). A p-value of $<0.05$ was considered statistically significant.

\section{Ethics statement}

This study was approved by the Institutional Review Board of Daegu Catholic University Medical Center (IRB No. CR-18162), and informed consent was waived due to the retrospective nature of the study.

\section{RESULTS}

The number of institutions participating in the Continuous Quality Improvement program of the Korean Society of Cytopathology (KSC) has gradually increased from 196 in 2007 to 206 in 2017. The results of diagnostic accuracy and sample adequacy evaluation have been reflected in the proficiency assessment of the cytopathology area within the proficiency evaluation program, Pathology Division, Korean Society of Pathologists' Quality Assurance Committee.

\section{Statistics of cytopathology cases}

The total number of cytology results exceeded 6.1 in 2008, 7.7 million in 2011, 8.4 million in 2014, and 9.3 million in 2017. The number of GYN cytology results in 2008 was 4.34 million; in 2011, 5.63 million; in 2013, 6.18 million; and in 2017, 7.51 million. In 2013, of the number of total cytology results, the number of GYN cytology was 6.18 million, which accounted for $80.3 \%$; non-GYN cytology, 1.06 million, $13.8 \%$; and FNA cytology, 450,000, 5.9\%. In 2017, of the number of total cytology, the number of GYN cytology was 7.51 million, accounting for $80.3 \%$; non-GYN cytology, 1.54 million, $16.5 \%$; and FNA cytology, about $300,000,3.2 \%$. Of the number of non-GYN cytology results, the number of respiratory cytology samples, including sputum, accounted for the largest number, followed by urine and then body fluid. In relation to the FNA, thyroid gland FNA was the highest at $68.4 \%$. In 2013 , the number of GYN LBC was 1.65 million, or $26.8 \%$ of the total GYN cytology results. In 2017, 1.99 million LBC were performed, accounting for $21.3 \%$ of the number of total cytology results. Of them, the number of GYN LBC was 1.52 million, $20.3 \%$ of the total GYN cytology results, and the number of non-GYN LBC was 340,000, 22.3\% of the total non-GYN cytology results. The total number of FNA tests implemented as LBC was 120,000 , accounting for $40.4 \%$ of all FNA tests (Table 2).

\section{Statistics of GYN cytology}

In 2010, of the number of GYN cytology results, $61.1 \%$ were implemented by commercial laboratories; this frequency increase in 2011 to $73.2 \%$; in 2014 to $77.1 \%$; in 2015 to $77.9 \%$; and in 2017 to $79.2 \%$. Of all GYN cytology results, in $2009,95.9 \%$ were negative; $2.2 \%$ were atypical squamous cells of undetermined significance (ASC-US); $0.2 \%$ were atypical squamous cells cannot exclude high-grade squamous lesions (ASC-H); $0.9 \%$ were low-grade squamous intraepithelial le- 
sions (LSIL); $0.4 \%$ were high-grade squamous intraepithelial lesions (HSIL); $0.12 \%$ were squamous cell carcinomas (SQCC); and $0.02 \%$ were atypical glandular cells (AGC). In 2017, 95.1\% were negative; $3.3 \%$ were ASC-US; $0.2 \%$ were ASC-H; $0.7 \%$ were LSIL; $0.2 \%$ were HSIL; $0.02 \%$ were SQCC; and $0.06 \%$ were AGC. The atypical squamous cell/squamous intraepithelial lesion (ASC/SIL) ratio was 3.00 in 2013 and gradually increased to 3.82 in 2017 ( $\mathrm{p}=.011)$. The ASC/SIL ratio of commercial laboratories increased from 3.68 in 2013 to 4.95 in 2017 ( $\mathrm{p}=.004$ ). The ASC/SIL ratio of university hospitals increased from 1.70 in 2013 to 1.95 in $2017(\mathrm{p}=.042)$ (Table 3). With respect to concordance between GYN cytology and histology evaluated by each institution, in the year 2009, category $\mathrm{O}$ accounted for $78.3 \%$; category A for $11.6 \%$; category B for $8.6 \%$; and category $\mathrm{C}$ for $1.4 \%$. In 2013 , category $\mathrm{O}$ was $86.3 \%$; while categories $\mathrm{A}, \mathrm{B}$, and $\mathrm{C}$ were $8.9 \%, 4.0 \%$, and $0.8 \%$, respectively. In 2017, category $\mathrm{O}$ was $84.8 \%$; and categories $\mathrm{A}, \mathrm{B}$, and $\mathrm{C}$ were $11.0,3.7 \%$, and $0.5 \%$.

\section{External proficiency test}

The external proficiency tests for GYN cytology samples pro-

Table 2. Total cytology cases according to category and method in 2017

\begin{tabular}{llll}
\hline & \multicolumn{1}{c}{ GYN } & \multicolumn{1}{c}{ Non-GYN } & \multicolumn{1}{c}{ FNA } \\
\hline Total & $7,510,563$ & $1,539,926$ & 297,885 \\
CS & $5,984,869(79.7)$ & $1,196,103(77.6)$ & $177,679(59.6)$ \\
LBC & $1,525,694(20.3)$ & $343,823(22.4)$ & $120,206(40.4)$ \\
\hline
\end{tabular}

Values are presented as number (\%).

GYN, gynecology; FNA, fine needle aspiration; CS, conventional smear; LBC, liquid based cytology. vided by CQIKSC were analyzed based on category. As a result, in 2014, category $\mathrm{O}$ represented $81.0 \%$ of the results; category A, $12.4 \%$; category B, $5.1 \%$; and category C, $1.5 \%$. In 2017, category $\mathrm{O}$ comprised $78.1 \%$; category A, $13.2 \%$; category B, $6.5 \%$; and category $\mathrm{C}, 2.2 \%$. With respect to diagnostic consistency between non-GYN cytology of respiratory, urine, and body fluid samples, in 2014, category $\mathrm{O}$ was $91.1 \%$; category A, 5.5\%; category B, 2.4\%; and category C, 1.0\%. In 2017, category $\mathrm{O}$ was $88.0 \%$; category $\mathrm{A}, 8.7 \%$; category $\mathrm{B}, 2.5 \%$; and category $\mathrm{C}, 0.8 \%$. The FNA cytology in 2014 showed category O, $99.0 \%$; category A, $0.5 \%$; category B, $0.5 \%$; and category C, $0.0 \%$. In 2017, these values changed to category O, 95.8\%; category A, 2.1\%; category B, $1.3 \%$; and category C, $0.8 \%$ (Fig. 1).

\section{GYN cytology specimen adequacy evaluation}

In 2013, inadequate results for GYN cytology specimens were found in eight of the 77 participating university hospitals (10.4\%), eight of 77 participating general hospitals (10.4\%), and one of the 35 included commercial laboratories (2.9\%). In 2017 , two of 79 university hospitals (2.5\%) and two of 74 general hospitals $(2.7 \%)$ were inadequate. The total number of inadequate results decreased from 2013, but increased to three among the 30 commercial laboratories (10.0\%). However, in the samples with an unsatisfactory institution-specific diagnostic ratio among all GYN diagnoses in 2017, university hospitals had a proportion of $0.39 \%$; general hospitals, $1.9 \%$; and commercial laboratories, as low as $0.07 \%$.

Table 3. Changes of abnormal gynecologic cytology diagnostic criteria according to laboratory category from 2012 to 2017

\begin{tabular}{|c|c|c|c|c|c|c|c|}
\hline Year & Laboratory category & No. of ASCs & ASC rate (\%) & No. of SILS & SIL rate (\%) & ASC/SIL ratio & No. of carcinomas \\
\hline \multirow[t]{4}{*}{2012} & $\mathrm{UH}$ & 27,143 & 3.13 & 15,960 & 1.84 & 1.70 & 1,323 \\
\hline & $\mathrm{GH}$ & 19,460 & 3.17 & 7,627 & 1.24 & 2.55 & 571 \\
\hline & $\mathrm{CL}$ & 124,219 & 2.99 & 33,737 & 0.81 & 3.68 & 953 \\
\hline & Total & 170,822 & 3.03 & 57,324 & 1.02 & 2.98 & 2,847 \\
\hline \multirow[t]{4}{*}{2014} & $\mathrm{UH}$ & 29,624 & 3.41 & 17,034 & 1.96 & 1.74 & 1,299 \\
\hline & $\mathrm{GH}$ & 19,703 & 2.90 & 7,631 & 1.12 & 2.58 & 658 \\
\hline & $\mathrm{CL}$ & 183,076 & 3.50 & 43,511 & 0.83 & 4.21 & 887 \\
\hline & Total & 232,403 & 3.42 & 68,176 & 1.00 & 3.41 & 2,844 \\
\hline \multirow[t]{4}{*}{2016} & $\mathrm{UH}$ & 31,944 & 3.65 & 17,771 & 2.03 & 1.80 & 1,276 \\
\hline & $\mathrm{GH}$ & 18,867 & 2.95 & 6,958 & 1.09 & 2.71 & 419 \\
\hline & $\mathrm{CL}$ & 219,749 & 3.77 & 49,407 & 0.85 & 4.45 & 843 \\
\hline & Total & 270,560 & 3.68 & 74,136 & 1.01 & 3.65 & 2,538 \\
\hline \multirow[t]{4}{*}{2017} & UH & 41,371 & 3.82 & 21,173 & 1.96 & 1.95 & 1,188 \\
\hline & $\mathrm{GH}$ & 14,489 & 3.04 & 5,616 & 1.18 & 2.58 & 210 \\
\hline & $\mathrm{CL}$ & 204,737 & 3.44 & 41,374 & 0.70 & 4.95 & 622 \\
\hline & Total & 260,597 & 3.47 & 68,163 & 0.91 & 3.82 & 2,020 \\
\hline
\end{tabular}

ASC, atypical squamous cell; SIL, squamous intraepithelial lesion; UH, university hospital; GH, general hospital; CL, commercial laboratories. 
Trend of the frequency of category $\mathrm{C}$

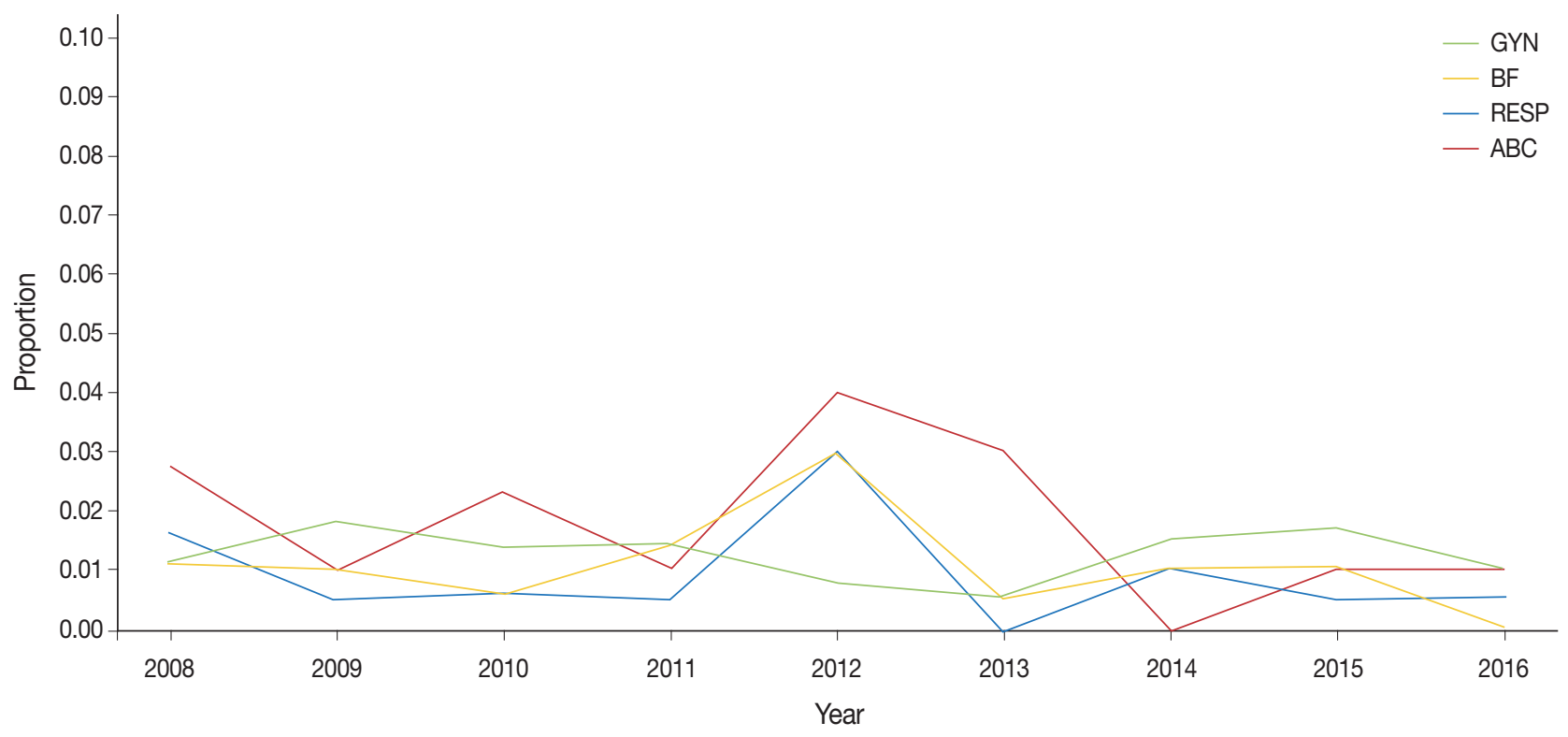

Fig. 1. Trend of frequency of category $\mathrm{C}$ in diagnostic accuracy evaluation (external quality control). GYN, gynecology; BF, body fluid; RESP, respiratory; $\mathrm{ABC}$, aspiration cytology.

\section{Quality assurance slide submission evaluation}

Slides were submitted to the CQIKSC in the final round each year to be reviewed by quality assurance committee members in the following year for suitability as quality assurance slides. Slides that had been judged to be appropriate were used for proficiency test 2 years later. Of the 1,035 slides submitted in 2016, 473 were utilized for proficiency tests in 2018. In 2013, inappropriate slides from university or general hospitals accounted for $39.2 \%$ of the total not used for testing, and that at commercial laboratories was $60.0 \%$; these numbers declined to $24.6 \%$ and $41.0 \%$ in 2016, respectively

\section{DISCUSSION}

The number of institutions participating in the Continuous Quality Improvement program managed by the CQIKSC has increased each year to include most of the institutions that practice cytopathology nationwide. The Continuous Quality Improvement program includes the degree of inconsistency between cytologic statistics and diagnostic accuracy, sample appropriateness, and quality assurance slide submission evaluation.

The cytopathologic quality assurance checklist was independently initiated by the KSC. However, in 2007, it was moved into the cytopathologic area of the checklist for the "pathology division's quality control" implemented by the Korean Society of Pathologists. Nevertheless, modification, improvement, and evaluation of any details are performed by CQIKSC. The checklist addresses quality assurance in cytopathologic laboratories, personnel management, operating manuals, test equipment, test methods, reporting systems, environment, safety measures, etc.

In the initial period after the LBC method was introduced in GYN cytology, the number and ratio of LBC tended to increase continuously; however, the ratio decreased to $21.4 \%$ in 2017 from $26.8 \%$ in 2013 . However, the total number of GYN LBC slightly decreased to 1.52 million in 2017 from 1.65 million in 2013. It seems that the ratio of total GYN samples submitted to commercial laboratories continuously increased, as most national cancer screening GYN samples are referred to these entities. LBC is not being used for the national cancer screening project. LBC, however, was found to account for a larger portion of the FNA test compared to GYN cytology or non-GYN cytology in 2017. It seems that LBC is largely introduced in FNA tests for the thyroid gland $[5,6]$. Nonetheless, further general investigation on the issue is necessary.

Regarding the statistics on gynecologic cytology, the ratio of SQCC decreased from $0.12 \%$ in 2009 to $0.02 \%$ in 2017 . AGC accounted for $0.02 \%$ in 2009 and increased to $0.06 \%$ in 2017. Compared to 2009, the number of total tests surged in 2017, while the ratio of inappropriate results remained relatively constant. These results are correlated with the positive effects of increased frequency of regular screening under the national cancer prevention project and declining cancer incidence with socio- 
economic improvement. This supports the view that domestic cervical SQCC incidence reduces cancer registration results. The data are also consistent with an increasing incidence of adenocarcinoma [7].

Given that the purpose of GYN cytology is primarily to detect SQCC in the early stage, the initial lesion, ASC (ASC-US and ASC-H), is most important. Many institutions have aimed to maintain the ASC diagnosis and ASC/SIL ratios within appropriate levels, which means that SIL lesions reflect low inter-observer variation and are more objective than ASC for maintaining ASC objectivity [8-11]. In the initial period after 2009, the ASC/ SIL ratio was maintained at 2.00 or under but increased to 3.00 in 2013 and then further to 3.82 in 2017 . Commercial laboratory ASC/SIL rate $(2013,3.68 ; 2017,4.95)$ is growing more than that of university hospitals $(2013,1.70 ; 2017,1.95)$. Compared with university hospitals commercial laboratories rarely implement simultaneous biopsy, resulting in relatively high ASC and low SIL ratios. This finding will require further review.

The histologic diagnostic accuracy of each institution's GYN cytology was not significant. Most pathology departments lacked the necessary resources such as personnel and computer systems, making it difficult to perform and maintain statistical processes for diagnostic consistency. Moreover, there were no criteria on whether tissue sample subject comparison was based on punch biopsy, cone biopsy, or hysterectomy. There was no mention on the time difference between cytology and histology. The criteria for assessment of diagnostic accuracy about GYN cytology were different according to time, limiting comparison of category ratios over years. For quality improvement of each institution, a previous GYN cytology slide review seems a good method, in addition to cytology and histology correlation diagnostic consistency analysis. Reviewing $10 \%$ of slides under ASC and reviewing high-risk groups is recommended [12].

With respect to the external proficiency test, most of the institutions showed a lower diagnostic accuracy rate of GYN cytology compared with non-GYN or FNA cytology. This is likely because, compared with other samples, GYN cytology and diagnosis structure are more complicated and difficult. Some other countries divide the diagnostic codes of GYN cytology into three categories of inadequate sample, negative or reactive lesion, and epithelial cell abnormal lesion. Minor differences within a category are defined as minor discrepancies and differences between categories as major discrepancies to determine false positivity and false negativity. In this situation, it seems necessary to improve the discrepancy grade system as defined by the CQIKSC.

The sample adequacy evaluation that has been implemented since 2013 is related to the diagnosis rate of inadequate samples. The percentage of inadequate samples in university hospitals decreased in 2017, compared with 2013, while the percentage in commercial laboratories increased. Regarding the finding that commercial laboratories had a very low percentage of unsatisfactory diagnosis in GYN cytology compared to university and general hospitals, an important factor could be that commercial laboratories are pushed to maintain higher thresholds for 'inappropriate/inadequate samples' because they rather not offend the referring clinicians by implying that they had not done well in collecting the samples.

The most urgent and necessary aspect observed while organizing the quality control results was that, if participating institutions utilized a common program for internal and external quality assurance, they would efficiently make use of the quality assurance results. If a quality assurance program compatible for each institution is developed by the Society or Committee and provided to institutions for quality control data organization, it would be very helpful for future advancement of cytopathology. In addition to such a system, other programs that need to be developed include quality assurance on the human papillomavirus test and quality assurance on automatic screening equipment for GYN cytology.

The KSC, in cooperation with the CQI program, has developed a computer program and plan to utilize it beginning in 2018. This more advanced and efficient quality improvement program is expected to play an advanced role in determining the foundation for cancer prevention and treatment. The KSC quality improvement program has continuously developed since 1995, comprising about 206 participating institutions. Four evaluations are implemented each year. Since 2008, factual surveys in cytopathology have been integrated into the "pathology division's quality control" program. However, inspection is currently ongoing. With the national cancer prevention project expansion, an increasing number of GYN cytology tests are being implemented by commercial laboratories. Although the number of LBC increased in various areas, its proportion in GYN cytology tended to gradually decrease. In GYN cytology, the proportion of SQCC diagnoses is decreasing, whereas the proportion of AGC is rising. Compared with university or general hospitals, commercial laboratories tend to show a lower rate of unsatisfactory diagnoses in GYN cytology with a higher ASC/SIL frequency. External diagnostic accuracy remains high. It seems that more advanced and higher-quality upgrades would be achieved when the computerized cytopathologic quality assurance program launches in 2018. The researchers also hope for expedited devel- 
opment of quality assurance programs on human papillomavirus and automatic screening equipment for GYN cytology.

\section{ORCID}

Yoo-Duk Choi: https://orcid.org/0000-0002-4385-1759

Hoon-Kyu Oh: https://orcid.org/0000-0001-8793-1948

Su-Jin Kim: https://orcid.org/0000-0001-5954-5281

Kyung-Hee Kim: https://orcid.org/0000-0003-0214-0296

Yun-Kyung Lee: https://orcid.org/0000-0001-6821-8653

Bo-Sung Kim: https://orcid.org/0000-0002-2344-7230

Eun-Jeong Jang: https://orcid.org/0000-0003-1482-5022

Yoon-Jung Choi: https://orcid.org/0000-0002-5701-8864

Eun-Kyung Han: https://orcid.org/0000-0002-7521-5095

Dong-Hoon Kim: https://orcid.org/0000-0001-5722-6703

Younghee Choi: https://orcid.org/0000-0002-3882-4000

Chan-Kwon Jung: https://orcid.org/0000-0001-6843-3708

Sung-Nam Kim: https://orcid.org/0000-0001-9235-7805

Kyueng-Whan Min: https://orcid.org/0000-0002-4757-9211

Seok-Jin Yoon: https://orcid.org/0000-0001-5879-1392

Hun-Kyung Lee: https://orcid.org/0000-0002-9802-5235

Kyung Un Choi: https://orcid.org/0000-0002-3848-1781

Hye Kyoung Yoon: https://orcid.org/0000-0003-0714-8537

\section{Author Contributions}

Conceptualization: YDC.

Data curation: KHK, YKL, EKH, YC, CKJ, KWM, SJY, HKL, KUC.

Formal analysis: SJK, BSK, DHK, SNK.

Investigation: HKO, EJJ, YJC.

Methodology: YDC, YJC.

Project administration: HKO, HKY.

Supervision: HKO, YJC, HKY.

Validation: YDC, HKO, YJC.

Visualization: HKO, DHK, CKJ.

Writing—original draft: YDC.

Writing—review \& editing: YDC, HKO, HKY.

\section{Conflicts of Interest}

C.K.J., the editor-in-chief of the Journal of Patbology and Translational Medicine, were not involved in the editorial evaluation or decision to publish this article. All remaining authors have declared no conflicts of interest.

\section{Funding}

This research was supported by the Korean Society for Cytopathology Grant No. 2017-01.

\section{REFERENCES}

1. Lee HK, Kim SN, Khang SK, Kang CS, Yoon HK. Quality control program and its results of Korean Society for Cytopathologists. Korean J Cytopathol 2008; 19: 65-71.

2. Oh EJ, Jung CK, Kim DH, et al. Current cytology practices in Korea: a nationwide survey by the Korean Society for Cytopathology. J Pathol Transl Med 2017; 51: 579-87.

3. Solomon D, Davey D, Kurman R, et al. The 2001 Bethesda System: terminology for reporting results of cervical cytology. JAMA 2002; 287: 2114-9.

4. Cibas ES, Ali SZ. The Bethesda System for Reporting Thyroid Cytopathology. Thyroid 2009; 19: 1159-65.

5. Fadda G, Rossi ED. Liquid-based cytology in fine-needle aspiration biopsies of the thyroid gland. Acta Cytol 2011; 55: 389-400.

6. Chong Y, Ji SJ, Kang CS, Lee EJ. Can liquid-based preparation substitute for conventional smear in thyroid fine-needle aspiration? A systematic review based on meta-analysis. Endocr Connect 2017; 6: 817-29.

7. Kim Y, Jun JK, Choi KS, Lee HY, Park EC. Overview of the National Cancer screening programme and the cancer screening status in Korea. Asian Pac J Cancer Prev 2011; 12: 725-30.

8. Confortini M, Carozzi F, Dalla Palma P, et al. Interlaboratory reproducibility of atypical squamous cells of undetermined significance report: a national survey. Cytopathology 2003; 14: 263-8.

9. Davey DD, Neal MH, Wilbur DC, Colgan TJ, Styer PE, Mody DR. Bethesda 2001 implementation and reporting rates: 2003 practices of participants in the College of American Pathologists Interlaboratory Comparison Program in Cervicovaginal Cytology. Arch Pathol Lab Med 2004; 128: 1224-9.

10. Nascimento AF, Cibas ES. The ASC/SIL ratio for cytopathologists as a quality control measure: a follow-up study. Am J Clin Pathol 2007; 128: 653-6.

11. Renshaw AA, Deschênes M, Auger M. ASC/SIL ratio for cytotechnologists: a surrogate marker of screening sensitivity. Am J Clin Pathol 2009; 131: 776-81.

12. Krieger P, Naryshkin S. Random rescreening of cytologic smears: a practical and effective component of quality assurance programs in both large and small cytology laboratories. Acta Cytol 1994; 38: 291-8. 